\title{
Computational Simulation of Bray-Liebhafsky (BL) Oscillating Chemical Reaction
}

\author{
Jie Ren, Jin Zhang Gao*, Wu Yang \\ Chemistry \& Chemical Engineering College, Northwest Normal University, \\ Lanzhon, 730070, PR China
}

Received $19^{\text {th }}$ January 2008; accepted $3^{\text {rd }}$ April 2008

\begin{abstract}
The computational simulation of the Bray-Liebhafsky (BL) oscillating chemical reaction by differential kinetic methodology is carried out in this work. According to the mechanism of Treindl and Noyes involving 10 reaction steps, the changes of the concentrations of $\mathrm{I}_{2}$ and $\mathrm{O}_{2}$ in solution are simulated. When the control parameters are $\alpha=0.55, \beta=0.2882$ and $\delta<0.6$, the differential equations present periodic solution, and the oscillations can be observed in $150 \mathrm{~min}$. If $\alpha, \beta$ and $\delta$ are taken as the control parameters, respectively, the bifurcation points would be observed in the processes of control parameters, changing successively with the critical values of $\alpha=0.55, \beta=$ 0.2882 , and $\delta=0.6$. The acidity of solution on the nonlinear phenomena is also investigated in detail.
\end{abstract}

Keywords: computational simulation, Bray-Liebhafsky (BL) oscillating chemical reaction, differential kinetic methodology, bifurcation.

\section{Introduction}

A large number of oscillating chemical reactions have been investigated both experimentally and theoretically [1-4]. It is generally accepted that at least two different conditions are necessary for the occurrence of chemical oscillations in a homogeneous chemical system: (1) the system should be far from thermodynamic equilibrium, and (2) the corresponding kinetic equations must be nonlinear. The first example of homogeneous oscillating chemical reaction was reported by Bray [5] after studying iodate-catalyzed decomposition of hydrogen peroxide in acidic medium over 80 years. The oscillating phenomena and the various component reactions were examined extensively by Bray and

\footnotetext{
* Corresponding author. E-mail address: jzgao@nwnu.edu.cn
} 
Liebhafsky, and the Bray-Liebhafsky (BL) oscillating chemical reaction was proposed. According to references [6,7], microwaves and light have obvious effect on the BL reaction. The overall chemical change in the BL system is described as progress (A):

$$
2 \mathrm{H}_{2} \mathrm{O}_{2}(\mathrm{aq}) \rightarrow 2 \mathrm{H}_{2} \mathrm{O}(\mathrm{aq})+\mathrm{O}_{2}(\mathrm{~g})
$$

The concentration of iodine and the rate of oxygen gas evolution change periodically in this system. The main reason of this phenomena results from the dual effects of hydrogen peroxide in the whole process. That is to say, hydrogen peroxide acts not only as the oxidant but also as the reductant in the system. The process $(A)$ is assumed to be the net result of processes $(B)$ and $(C)$ :

$$
\begin{gathered}
2 \mathrm{IO}_{3}{ }^{-}+5 \mathrm{H}_{2} \mathrm{O}_{2}+2 \mathrm{H}^{+} \rightarrow \mathrm{I}_{2}+5 \mathrm{O}_{2}+6 \mathrm{H}_{2} \mathrm{O} \\
\mathrm{I}_{2}+5 \mathrm{H}_{2} \mathrm{O}_{2} \rightarrow 2 \mathrm{IO}_{3}^{-}+2 \mathrm{H}^{+}+4 \mathrm{H}_{2} \mathrm{O}
\end{gathered}
$$

Processes (B) and (C) are not the reverse of each other and both proceed with negative Gibbs free energy $(\Delta G<0)$ [8]. If iodate was indeed acting only as a catalyst, the total concentration of iodate would be constant and the total chemical change of the processes (B) and (C) should be equal during any extended period of time. However, one process might be faster than the other during a short interval. Processes (B) and (C) alternately dominate over the whole system and result in the concentration of iodine and the rate of oxygen gas evolution change periodically [9-12].

Although the overall stoichiometry of the BL reaction seems to be simple, it is not easy to describe the mechanism. The difficulty lies on two aspects: (1) because of the system has few variables which could vary independently, it is difficult to observe the effects of each variable on the system; (2) it is very difficult to make clear the kinetic effect of oxygen on the BL reaction. In the BL reaction system oxygen yields continuously and its concentration in the solution is higher than that under normal conditions because of the existence of supersaturation phenomenon [13]. On one side, the oxygen is gaseous and it is too difficult to control it. On the other hand, the concentration of oxygen becomes supersaturated and the degree of super-saturation depends on the experimental conditions, such as the concentrations of reactants and temperature. There are several papers of the analysis of mechanism of $\mathrm{BL}$ reaction $[8,11,14,15]$. Matsuzaki [12] proposed a mechanism involving $\mathrm{I}_{3}{ }^{-}$; the question is how to verify it by experiment. Schmitz and Rooze [17] assumed that the HOI should be oxidized at fictitious equilibrium state; meanwhile, Sharma and Noyes [18] considered further that the free radical might be formed in the redox processes. Recently, Schmitz found that in an open reactor, where the flow rate was the variable parameter, different simple and complex oscillations and different routes to chaos were observed through numerical calculations [19]. So, up to now, there is no agreement about the BL reaction mechanism. 
This work simulates the nonlinear behavior of the BL reaction based on a model involving two variables. The bifurcations are observed in the process of changing parameters. The acidity of the solution on the nonlinear phenomena is investigated in detail.

\section{Mathematical model}

Treindl and Noyes [16] suggested the mechanism involving 10 individual steps in which the transport of oxygen from the supersaturated solution to atmosphere was a significant component of the overall mechanism of the batch BL system and radicals $\mathrm{I}$. and $\mathrm{IO}_{2}$. were involved in the whole process. The 10 individual steps are listed below:

$$
\begin{gathered}
\mathrm{IO}_{3}^{-}+\mathrm{I}^{-}+2 \mathrm{H}^{+} \rightarrow \mathrm{HIO}_{2}+\mathrm{HOI} \\
\mathrm{HIO}_{2}+\mathrm{I}^{-}+\mathrm{H}^{+} \rightarrow 2 \mathrm{HIO} \\
\mathrm{HOI}+\mathrm{I}^{-}+\mathrm{H}^{+} \rightleftarrows \mathrm{I}_{2}(\mathrm{aq})+2 \mathrm{H}_{2} \mathrm{O} \\
\mathrm{HOI}+\mathrm{H}_{2} \mathrm{O}_{2} \rightarrow \mathrm{I}^{-}+\mathrm{H}^{+}+\mathrm{O}_{2}(\mathrm{aq})+\mathrm{H}_{2} \mathrm{O} \\
\mathrm{I}^{-}+\mathrm{H}^{+}+\mathrm{H}_{2} \mathrm{O}_{2} \rightarrow \mathrm{HOI}+\mathrm{H}_{2} \mathrm{O} \\
\mathrm{I}_{2}(\mathrm{aq}) \rightarrow 2 \mathrm{I} \bullet \\
\mathrm{I}_{\bullet}+\mathrm{O}_{2}(\mathrm{aq}) \rightleftarrows \mathrm{IOO}^{\bullet} \\
\mathrm{IOO} \longrightarrow \mathrm{IO}_{2} \bullet \\
2 \mathrm{IO}_{2}+\mathrm{H}_{2} \mathrm{O} \rightarrow \mathrm{IO}_{3}^{-}+\mathrm{H}^{+}+\mathrm{HIO}_{2} \\
\mathrm{O}_{2}(\mathrm{aq}) \rightleftarrows \mathrm{O}_{2}(\mathrm{~g})
\end{gathered}
$$

In fact, process (B) is the sum of $\mathrm{R} 1, \mathrm{R} 2, \mathrm{R} 3, \mathrm{R} 4$ and $\mathrm{R} 10$. The species $\mathrm{IO}_{3}{ }^{-}$and $\mathrm{H}_{2} \mathrm{O}_{2}$ are the major reactants in the process (B), but they do not react with each other at a finite rate even though both are consumed in proportionate amounts in process (B), which takes place at a significant rate. The rate-determining step that initiates process (B) is step R1. $\mathrm{I}^{-}$is the principal reactant in step $\mathrm{R} 1$ and it is formed by rapid hydrolysis of the $\mathrm{I}_{2}$ product. The existence of autocatalytic process is one of the necessary conditions for the occurrence of oscillations. In the BL system the process (B) is autocatalytic.

Process (C), in which the iodine is oxidated to iodate, is even more complicated than autocatalytic process (B). The rate-determining step in process $(C)$ is step R1. Zimmerman [20] found that in degassed iodine atoms recombined with each other in one tenth of a second. However, in the presence of air, iodine atoms would persist for almost a minute. So iodine might be stabilized by oxygen in water. 
According to the mechanism proposed by Treindl and Noyes, this work simulates the nonlinear behavior of the BL reaction involving two variables. The variables indicate the concentrations of the components.

Table 1. Identification of symbols for variables and parameter.

\begin{tabular}{||c|c||}
\hline$X$ & $\mathrm{I}_{2}(\mathrm{aq})$ \\
\hline $\mathrm{Y}$ & $\mathrm{O}_{2}(\mathrm{aq})$ \\
\hline $\mathrm{A}$ & $\mathrm{IO}_{3}{ }^{-}$ \\
\hline $\mathrm{B}$ & $\mathrm{O}_{2}(\mathrm{eq})$ \\
\hline $\mathrm{C}$ & $\mathrm{H}_{2} \mathrm{O}_{2}$ \\
\hline
\end{tabular}

The acidity of the solution is an important factor for the nonlinear behavior, but the more of variables in the model, the more difficulty of calculation. Thus, in this paper, we neglect the acidity of solution. According to steps R6 and R7 the following equations can be obtained:

$$
\begin{gathered}
\frac{d\left[I_{2(a q)}\right]}{d t}=v_{R 3}-\frac{1}{2} k_{R 7}[I \cdot]\left[O_{2(a q)}\right] \\
\frac{d\left[O_{2(a q)}\right]}{d t}=k_{R 4}\left[H_{2} O_{2}\right][H O I]-k_{R 10}\left\{\left[O_{2(a q)}\right]-\left[O_{2(e q)}\right]\right\}
\end{gathered}
$$

where $k$ is the rate constant of each reaction, respectively, and $v_{R 3}$ is the general rate constant of reversible step R3. And step R1 is the rate-determining step in process (B).

$$
v_{R 3}=\frac{1}{2} k_{R 1}\left[I O_{3}^{-}\right]\left[I^{-}\right]
$$

If $K_{d}$ and $K_{h}$ are the dissociation equilibrium constants and hydrolysis equilibrium constants of $\mathrm{I}_{2}$, respectively, then, the following equations can be gained:

$$
\begin{gathered}
K_{d}=[I \cdot]^{2} /\left[I_{2(a q)}\right] \\
K_{h}=\left[H^{+}\right]\left[I^{-}\right][H O I] /\left[I_{2(a q)}\right]
\end{gathered}
$$

It can be seen from equation (5) that, if the concentration of $\left[\mathrm{I}^{-}\right]$was proportional to $X^{\alpha}$, then the concentration of HOI would be proportional to $X^{1-\alpha}$. If $k_{1}, k_{2}, k_{3}$ and $k_{4}$ were empirical rate constants for a particular system, then the above equations could be combined to generate equation (6) as the fundamental equations of the model: 


$$
\begin{aligned}
& \frac{d X}{d t}=k_{1} A X^{\alpha}-k_{2} X^{0.5} Y \\
& \frac{d Y}{d t}=k_{3} C X^{1-\alpha}-k_{4}(Y-B)
\end{aligned}
$$

The above differential equations show the relationship of concentrations with time among the components in the system, and concentration and time are dimension variables and parameters. So the above differential equations must be transformed into dimensionless equations before resolving, the concentrations $X$ and $Y$, the time $t$ and the rate constants also should be transformed into dimensionless variables and parameters. Here, these transformations involve replacing $X, Y$ and $t$ in the above equation by $x, y, \tau, \beta$ and $\delta$, which are defined as

$$
\begin{aligned}
& x=X\left[\frac{\left(k_{2} A\right)^{2}}{k_{2} k_{3} C}\right]^{-\frac{2}{5-6 \alpha}}, \quad y=Y \frac{k_{2}}{k_{1} A}\left[\frac{\left(k_{1} A\right)^{2}}{k_{2} k_{3} C}\right]^{-\frac{1-2 \alpha}{5-6 \alpha}}, \tau=t\left(k_{2} k_{3} C\right)^{0.5}\left[\frac{k_{1} A}{k_{2} k_{3} C}\right]^{-\frac{2 \alpha-1}{2(5-6 \alpha)}}, \\
& \delta=k_{4} \frac{1}{\left(k_{2} k_{3} C\right)^{0.5}}\left[\frac{k_{1} A}{k_{2} k_{3} C}\right]^{\frac{2 \alpha-1}{2(5-6 \alpha)}}, \quad \beta=B \frac{k_{2}}{k_{1} A}\left[\frac{\left(k_{1} A\right)^{2}}{k_{2} k_{3} C}\right]^{-\frac{1-2 \alpha}{5-6 \alpha}} \circ
\end{aligned}
$$

After the dimensionless transformation, the differential equations become as follows:

$$
\begin{aligned}
& \frac{d x}{d \tau}=x^{\alpha}-x^{0.5} y \\
& \frac{d y}{d \tau}=x^{1-\alpha}-\delta(y-\beta)
\end{aligned}
$$

Double-precision computations are performed on a personal computer and the mathematic 5.0 is used in all the simulations. The temporal concentrations of reaction system are calculated by Gear method. The precision of calculation is more than 20 bit validity numerals and the simulation time is 150 minutes. The differential equations are resolved and the graphs of $x$ - $\tau$ and $y$ - $\tau$ are obtained.

\section{Results and discussion}

\section{Results}

Fig. 1 shows the simulation results of the BL oscillating chemical system. In the batch system, the concentration of iodine and the product rate of oxygen change periodically, and the amplitude damped but the period hold the line. The oscillations can maintain 150 minutes. Parameters $\alpha, \beta$ and $\delta$ affect the nonlinear behaviors obviously, and it will be discussed later. 

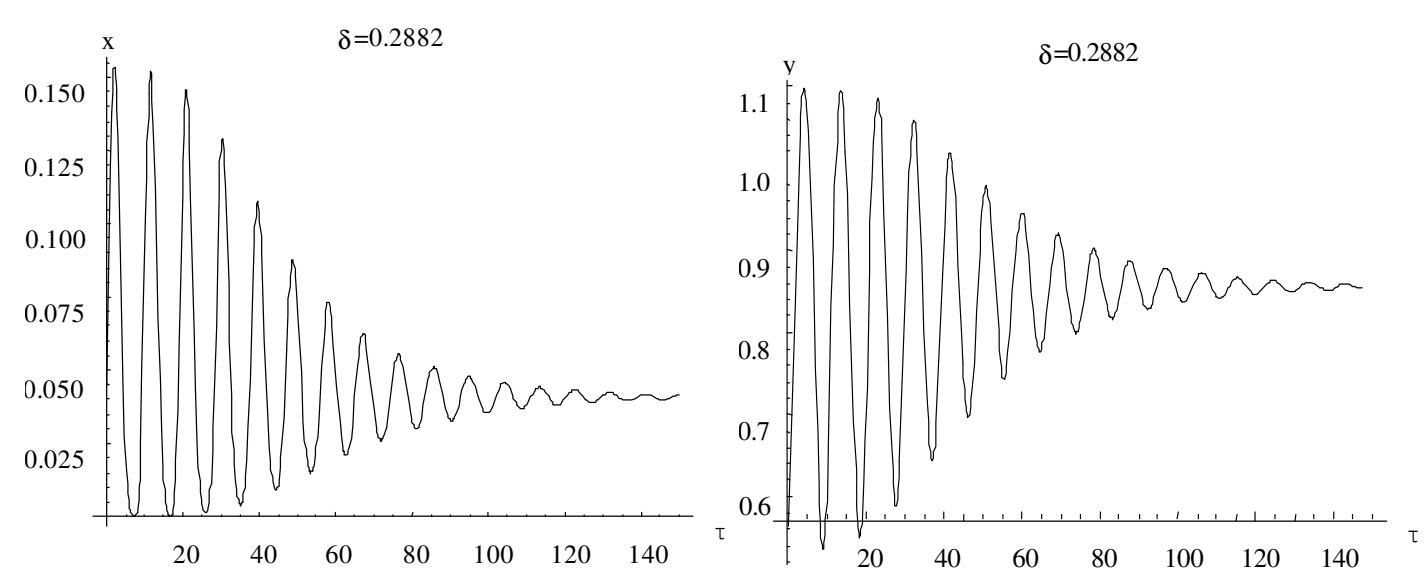

Figure 1. Simulation of the BL oscillations obtained by numerical integration with the differential equations ( $\alpha=0.55, \beta=0.03, \delta=0.2882$ ).

\section{Discussion}

\section{Effect of $\alpha$ on BL nonlinear behavior}

Equation (7) indicates that the value of $\alpha$ should be in the range of $0-1$. The simulation results showed that the number of period increased with increasing the $\alpha$ value in the range of $0-0.55$. That is to say, the larger $\alpha$ in this range, the slower decayed of oscillation in the system. In addition, the maximal concentrations of both $\mathrm{I}_{2(\mathrm{aq})}$ and $\mathrm{O}_{2(\mathrm{aq})}$ are decreased with the increase of $\alpha$ (see Fig. 2), where the value of $\alpha$ indicates the hydrolysis degree of $I_{2(a q)}$ in the solution. As mentioned above, the change of BL oscillating chemical could be divided into two processes (B) and (C); the $\mathrm{H}_{2} \mathrm{O}_{2}$ is oxidated by iodate to $\mathrm{I}_{2}$ in process (B) and $\mathrm{I}_{2}$ goes back to $\mathrm{IO}_{3}{ }^{-}$in process $(\mathrm{C})$. If the reaction rates of process (B) and process (C) were equal in any time, the system must achieve to chemical equilibrium state and no oscillation appeared. Just because of the reaction rate of process $(\mathrm{B})$ and process $(\mathrm{C})$, one is faster than the other at a certain time, so process (B) and process (C) are dominant on the oxidation and reduction processes alternatively, resulting in the oscillations of both $\mathrm{I}_{2}$ and $\mathrm{I}^{-}$ concentrations and the production rate of $\mathrm{O}_{2}$. If $\alpha$ was too small, then substantive $\mathrm{I}_{2}$ produced in process (B) translated into $\mathrm{IO}_{3}{ }^{-}$and process (C) dominated the system for a long time. It is too difficult to shift from process (C) to process (B), so the oscillations decay quickly and the system achieves to the chemical equilibrium state. On the contrary, if $\alpha$ was too large, then substantive $\mathrm{I}_{2}$ produced in process (B) hydrolyzes and the reaction rate of process (B) dominates the system and can not shift to the process $(\mathrm{C})$ and lead the system to reach the chemical equilibrium state, so no oscillation appears.

In order to investigate the effect of $\alpha$ on the nonlinear behavior of the BL oscillating chemical reaction, the value of $\alpha$ changes with step 0.01 in the range of 0.48-0.55 was researched in detail (see Fig. 3). Results show that the slight change of $\alpha$ brings to obviously change of the oscillation curves. More interesting is that when $\alpha=0.55$, the system oscillates in 150 minutes; but when $\alpha=0.56$, the differential equations described by the system have no solution in $\tau$ $=7$. From a mathematician view, if $\alpha$ was chosen as the control parameter and in 
the process of changing the qualitative character (there is stability) changes suddenly when $\alpha=0.55$. So it could be thought that the system could appear bifurcation when $\alpha=0.55$ and the critical value was 0.55 .
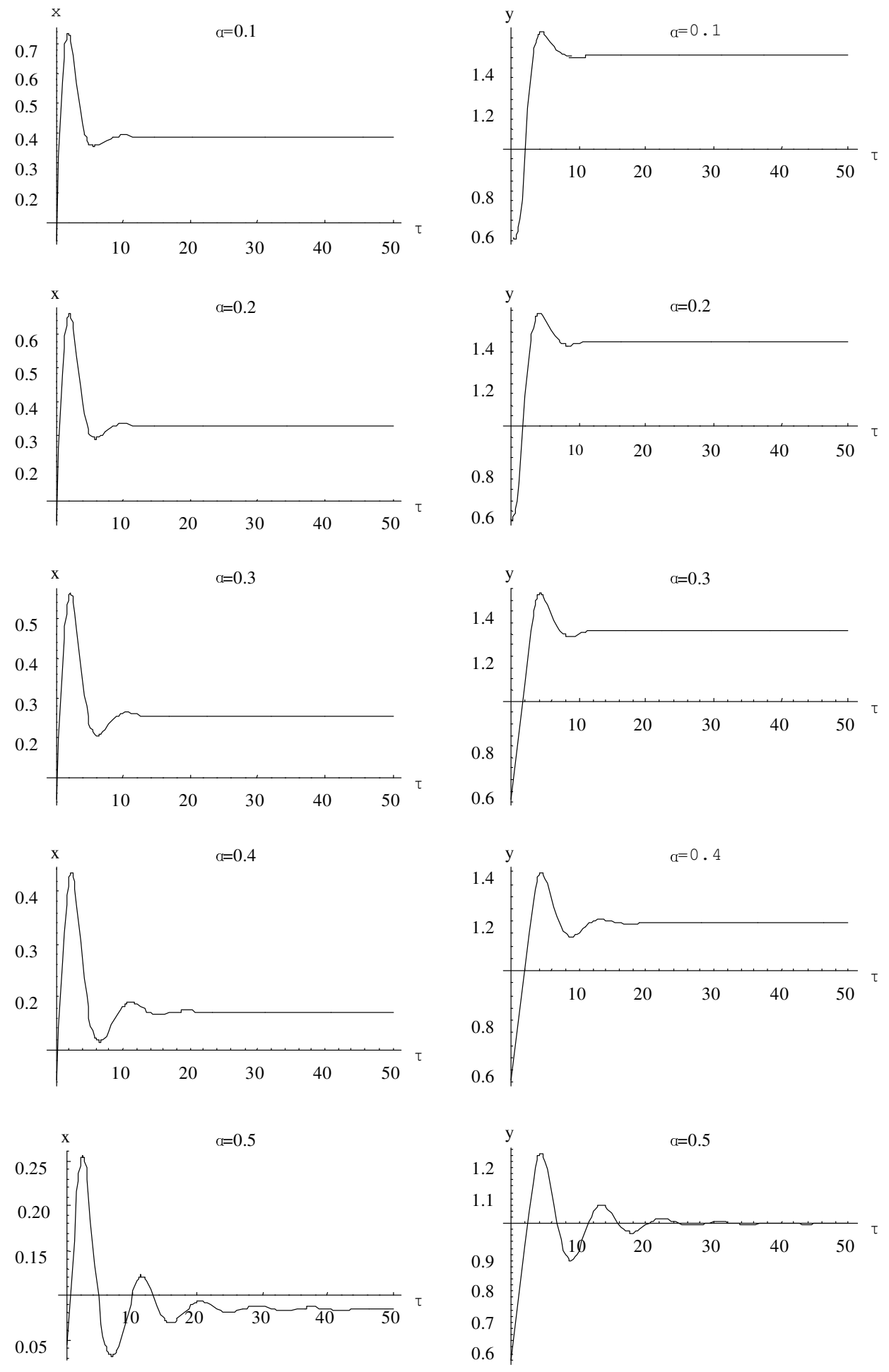

Figure 2. Effect of $\alpha$ in the range of $0-0.5$ on the oscillation $(\beta=0.03, \delta=0.2882)$. 

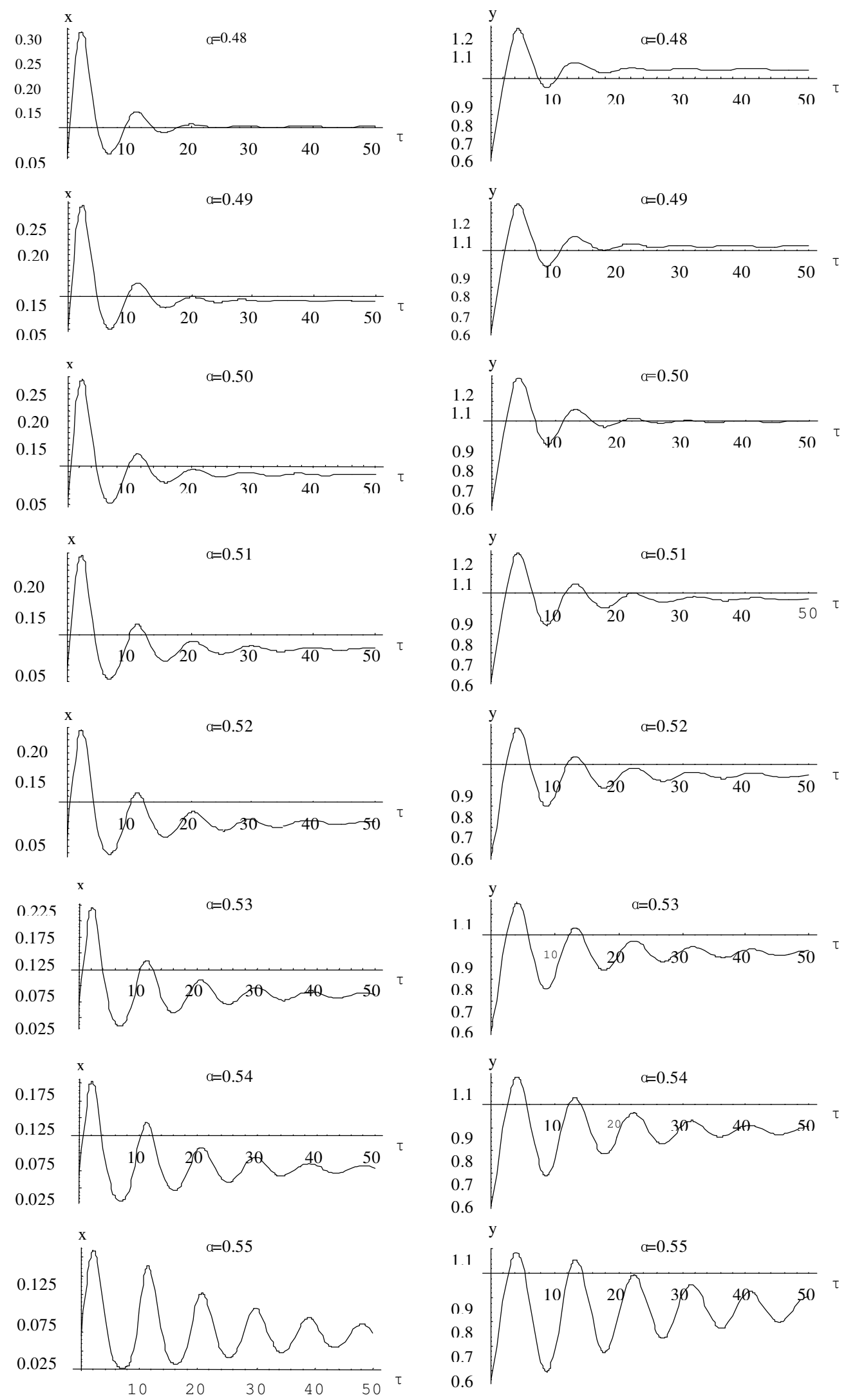

Figure 3. Effect of $\alpha$ in the range of $0.48-0.55$ on the oscillation $(\beta=0.03, \delta=0.2882)$. 

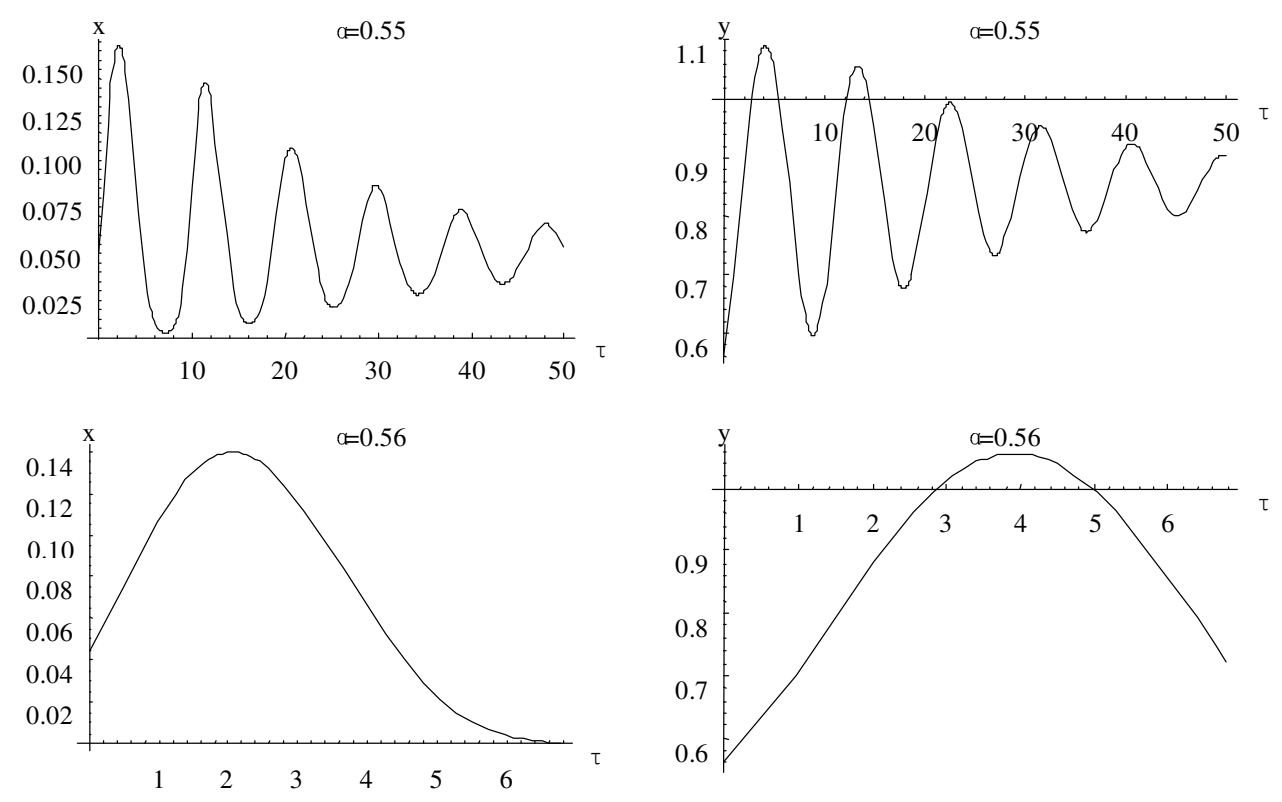

Figure 4. Comparison of $\alpha=0.55$ and $\alpha=0.56(\beta=0.03, \delta=0.2882)$.

\section{Effect of $\delta$ on BL nonlinear behavior}

From the definition of $\delta=k_{4} \frac{1}{\left(k_{2} k_{3} C\right)^{0.5}}\left[\frac{k_{1} A}{k_{2} k_{3} C}\right]^{\frac{2 \alpha-1}{2(5-6 \alpha)}}, \delta$ expresses the composition effects of the initial concentration of reactant, reaction rate constants and $\alpha$. Simulation results show that when $\delta \leq 0.2881$, the differential equations described by the system have no solution; when $\delta \geq 0.2882$, the differential equations have solution, and only for the value of $\delta$ in the range of $0.2882 \leq \delta \leq 1.1882$ the differential equations have periodic (or oscillating) solution, and the number of periodic solutions decreases with increasing $\delta$ in this range (see Fig. 5). When $\delta$ $\geq 1.1882$, the differential equations have solution but no periodic one. The same as $\alpha$, if $\delta$ was chosen as the control parameter and in the process of changing the qualitative character changes suddenly when $\delta=0.2881$. So the system appears bifurcation when $\delta=0.2881$ and the critical value is 0.2881 .

\section{Effect of $\beta$ on BL nonlinear behavior}

According to the simulation results, the effect of parameter $\beta$ on the oscillating curve is as not sensitive as $\alpha$ and $\delta$. When $\beta<0.6$, and the other parameters are consistent with the above discussion, the oscillation can be observed. The change of $\beta$ has little effect on the oscillation curve. There is no change between the curves of $\beta=0.03$ and $\beta=3 \times 10^{-9}$. In the same way, when $\beta=0.6$ the system appears bifurcation and the critical value is 0.6 . 

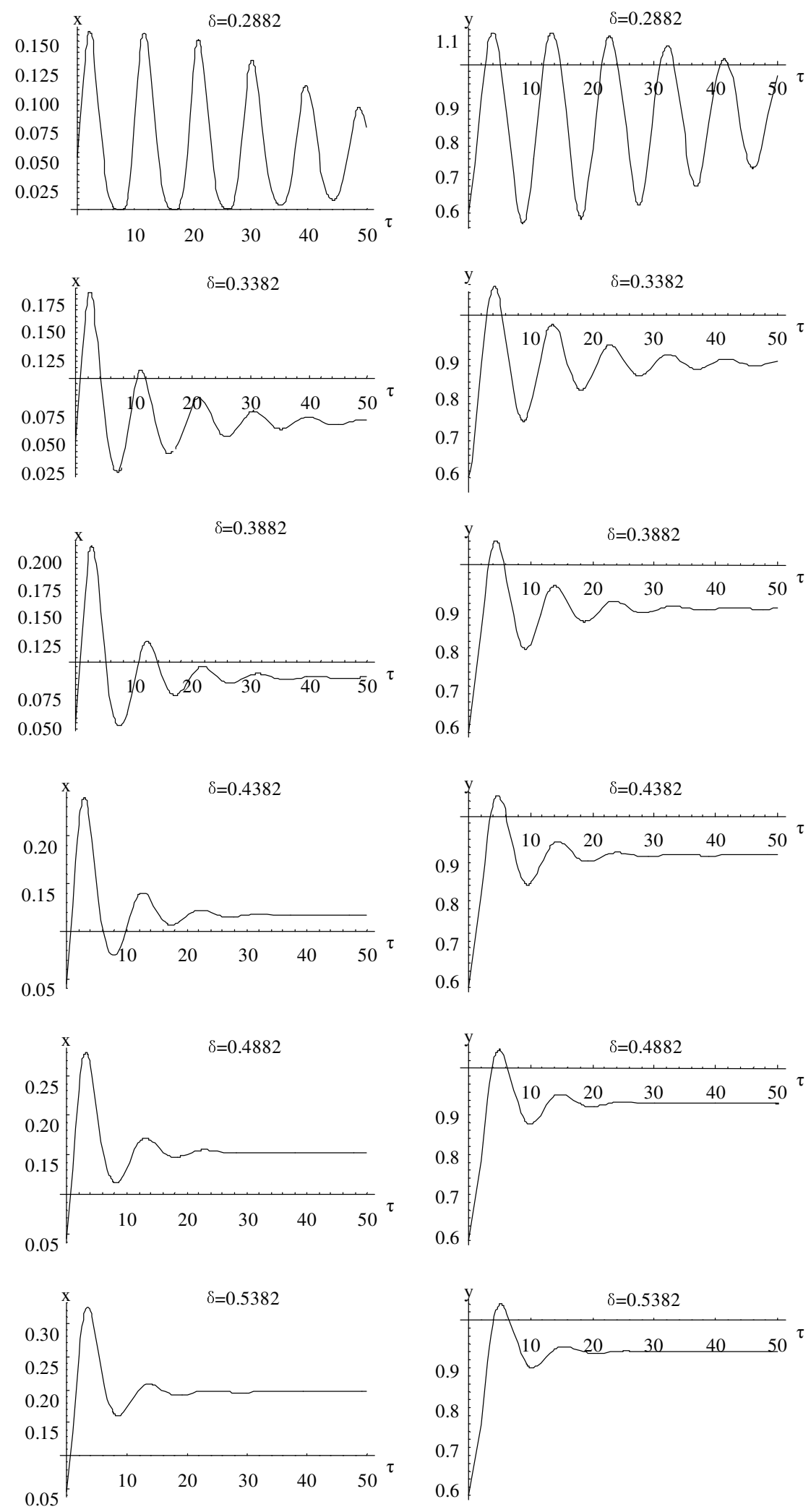

Figure 5. Effect of $\delta$ on the oscillation ( $\alpha=0.55, \beta=0.03$ ). 


\section{Effect of solution acidity on BL nonlinear behavior}

As pointed out in the introduction, the BL oscillating chemical reaction consists of processes (B) and (C). Not accounting for the reaction rate of them, the total iodine elementary in the system is equal to the initial concentration of iodate and keeps constant. The relative concentrations of $\mathrm{I}_{2}$ and $\mathrm{IO}_{3}{ }^{-}$depend on the acidity of the solution. According to equation (5), $\left[\mathrm{H}^{+}\right]\left[\mathrm{I}^{-}\right][\mathrm{HOI}]=K_{h}\left[I_{2(a q)}\right]$, if $\left[\mathrm{H}^{+}\right]$ is small $\left(\left[\mathrm{H}^{+}\right]=0.01 \mathrm{M}\right)$, then $\left[\mathrm{I}^{-}\right][\mathrm{HOI}]$ is larger [17] and the concentration of $\mathrm{I}_{2}$ increases even to saturation [18]. Noyes [8] reported a much larger formation of $\mathrm{I}_{2}$ at low than at high acidity. At lower acidity, formation of $\mathrm{I}_{2}$ cannot be oxidized to $\mathrm{IO}_{3}{ }^{-}$and no oscillation is observed. In addition, lower acidity corresponds to high value of $\alpha$. If the acidity of the solution is high $\left(\left[\mathrm{H}^{+}\right]=0.1 \mathrm{M}\right)$, the concentration of $\mathrm{I}_{2}$ would be much smaller than that in lower acidity, so it corresponds to higher value of $\alpha$ in the model. For $\alpha<0.5$, the mathematical simulation showed that equation (7) wouldn't be able of generating long-lasting oscillations. It is consistent with lower $\alpha$ and higher acidity. However, when the concentration of $\left[\mathrm{H}^{+}\right]=0.05 \mathrm{M}$, the system was alternately dominated, firstly by process (B) and then by process (C), resulting long-lasting oscillation. This situation corresponds to $\alpha$ slightly greater than 0.5 . This is the range of $\alpha$ values where oscillations appear in the simulation results reported in the above section.
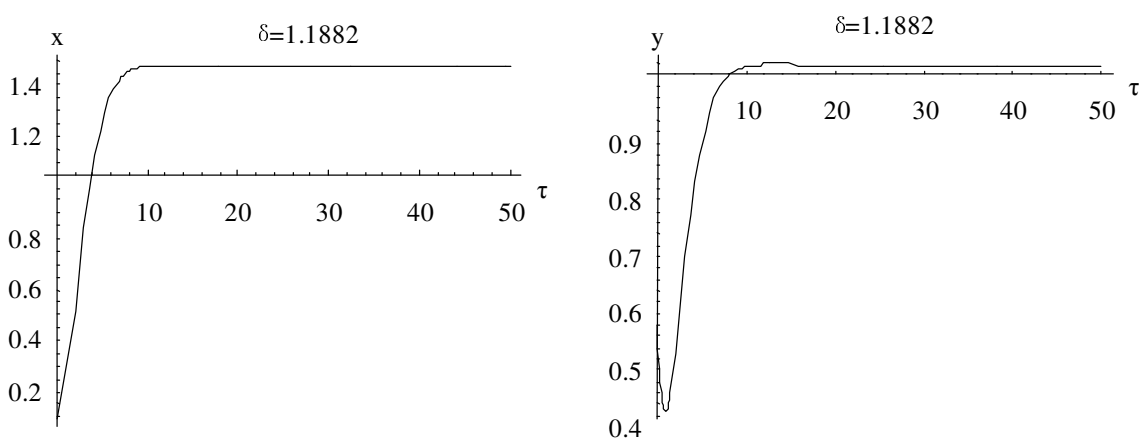

Figure 6. Curves of $\delta=1.1882(\alpha=0.55, \beta=0.03)$.

\section{Conclusion}

According to the mechanism proposed by Treindl and Noyes involving 10 reaction steps, the simulation of the BL oscillating chemical reaction by differential kinetic methodology was carried out in this work. In addition, the parameters of the differential equations were investigated in detail. The simulation results show that (1) when the variable parameters are $\alpha=0.55, \beta=$ 0.2882 and $\delta<0.6$, the differential equations present periodic solution, and the oscillation can be observed in $150 \mathrm{~min}$; (2) if $\alpha, \beta$ and $\delta$ were taken as the control parameters respectively, the bifurcations would be observed in the 
process with the critical values of $\alpha=0.55, \beta=0.2882$, and $\delta=0.6$; (3) the acidity of the solution is an important factor on the nonlinear phenomena. When the concentration of $\mathrm{H}^{+}$is about $0.05 \mathrm{M}$, that equivalent to $\alpha=0.5$, the oscillation can be observed.

\section{Acknowledgment}

The authors are grateful to the financial support in part by the National Natural Science Foundation (20475044), the Project of Excellent Teachers of Education Ministry (3070), and the project of KJCXGC-01 of Northwest Normal University, as well as the Gansu Key Lab of Polymer Materials, China.

\section{References}

1. V. Vukojević, S. Anić, Lj. Kolar-Anić, Phys. Chem. Chem. Phys. 4 (2002) 1276-1283.

2. J. Ren, J.Z. Gao, W. Yang, Comput. Visual. Sci. (2008) DOI 10.1007/s00791-008 -0092-2 (online first).

3. S. Anić, Lj. Kolar-Anić, E. Korös, React. Kinet. Catal. Lett. 61 (1997) 111116.

4. Lj. Kolar-Anić, Ž. Čupič, S. Anić, G. Schmitz, J. Chem. Soc. Faraday Trans. 93 (1997) 2147-2152.

5. W.C. Bray, J. Am. Chem. Soc. 43 (1921) 1262-1267.

6. D.R. Stanisavljev, A.R. Djordjević, V.D. Likar-Smiljanić, Chem. Phys. Lett. 423 (2006) 59-62.

7. S. Keki, G. Szekely, M.T. Beck, J. Phys. Chem. A 107 (2003) 73-75.

8. R.M. Noyes, L.V. Kalachev, R.J. Field, J. Phys. Chem. 99 (1995) 35143520.

9. P. Ševčík, K. Kissimonová, L. Adamčíková, J. Phys. Chem. A 104 (2000) 3958-3963.

10. F.G. Buchholtz, S. Broecker, J. Phys. Chem. A 102 (1998) 1556-1559.

11. I. Valent, L. Adamčíková, P. Ševčík, J. Phys. Chem. A 102 (1998) 75767579.

12. I. Matsuzaki, T. Nakajima, H.A. Liebhafsky, Faraday Symp. Chem. Soc. 9 (1974) 55-65.

13. P.G. Bowers, Ch. Hofstetter, C.R. Letter, R.T. Toomey, J. Phys. Chem. 99 (1995) 9632-9637.

14. E. David, R.M. Noyes, J. Phys. Chem. 83 (1979) 212 -220.

15. K. Kissimonová, I. Valent, L. Adamčíková, P. Ševčík, Chem. Phys. Lett. 341 (2001) 345-350.

16. L. Treindl, R.M. Noyes, J. Phys. Chem. 97 (1993) 11354-11362.

17. G. Schmitz, H. Rooze, Far from Equilibrum Synergetics, Springer-Verlly, Berlin, 1979, p51-56.

18. V. Sharma, R.M. Noyes, J. Am. Chem. Soc. 98 (1976) 4345-4361.

19. G. Schmitz, Lj. Kolar-Anić, S. Anić, J. Phys. Chem. A 110 (2006) 1036110368.

20. J. Zimmerman, R.M. Noyes, J. Phys. Chem. 18 (1950) 658-666. 